\title{
ApoE knockout mice expressing human matrix metalloproteinase-1 in macrophages have less advanced atherosclerosis
}

\author{
Vincent Lemaître, ${ }^{1}$ Timothy K. O’Byrne, ${ }^{1}$ Alain C. Borczuk, ${ }^{2}$ Yasunori Okada, ${ }^{3}$ \\ Alan R. Tall, ${ }^{1}$ and Jeanine D'Armiento ${ }^{1}$ \\ ${ }^{1}$ Division of Molecular Medicine, Department of Medicine, and \\ ${ }^{2}$ Department of Pathology, Columbia University College of Physicians and Surgeons, New York, New York, USA \\ ${ }^{3}$ Department of Pathology, Keio University, Tokyo, Japan \\ Address correspondence to: Jeanine D’Armiento, College of Physicians and Surgeons of Columbia University, \\ Department of Medicine, P\&S 8-401, 630 West 168th Street, New York, New York 10032, USA. \\ Phone: (212) 305-3745; Fax: (212) 305-5052; E-mail: jmd12@columbia.edu.
}

Received for publication February 15, 2000, and accepted in revised form April 16, 2001.

Matrix metalloproteinase-1 (MMP-1), or interstitial collagenase, has been hypothesized to contribute to the progression of the human atherosclerotic lesions by digesting the fibrillar collagens of the neointimal ECM. The apoE knockout (apoE0) mouse model develops complex atherosclerotic lesions, but mice do not possess a homologue for MMP-1. To provide an in vivo evaluation of the role of MMP-1 in atherogenesis, we created a transgenic mouse model that expresses this enzyme specifically in the macrophage, under the control of the scavenger receptor A (SCAV) enhancer/promoter. The MMP-1 transgenic mice were crossed into the apoE0 background and fed an atherogenic diet for 16-25 weeks. Surprisingly, the transgenic mice demonstrated decreased lesion size compared with control littermates. The lesions of the transgenic animals were less extensive and immature, with fewer cellular layers and a diminished content of fibrillar collagen. There was no evidence of plaque rupture. Our data suggest that remodeling of the neointimal ECM by MMP-1 is beneficial in the progression of lesions.

J. Clin. Invest. 107:1227-1234 (2001).

\section{Introduction}

Atherosclerosis, one of the major diseases of the Western world, is a complex inflammatory process that can profoundly affect the constitution of the vessel wall of major arteries (1). Atherosclerotic lesions are first characterized by the presence of inflammatory cells, mainly lipid-filled macrophages, forming fatty streaks in the neointima of the vessel. Evolution of the disease results in more complex lesions, containing necrotic cores, migrating smooth muscle cells, and macrophages amid prominent ECM (1-3). The mature atherosclerotic plaque is covered by a fibrous cap mainly composed of fibrillar collagens, elastin, and proteoglycans (2). Rupture of the fibrous cap leads to the liberation of highly thrombogenic molecules into the circulating blood. Subsequent thrombus formation often results in acute coronary syndromes, including lethal myocardial infarction $(1,4)$.

Recent observations suggest that enzymes belonging to the matrix metalloproteinase (MMP) family (5) are critically involved in matrix remodeling of the atherosclerotic lesions. These MMPs, including interstitial collagenase (MMP-1) (6,7), gelatinases A (MMP-2) (8) and B (MMP9) $(6,9,10)$, stromelysin-1 (MMP-3) $(6,11)$, matrilysin (MMP-7) (12), macrophage metalloelastase (MMP-12) (12), collagenase-3 (MMP-13) (13), and membrane-type MMP (MMP-14) (14), have been detected at the protein or mRNA level in the atherosclerotic lesions, but not in normal human vessels. Interstitial collagenase (MMP-1), together with stromelysin-1, was also shown to be constitutively expressed by macrophage foam cells of rabbit aortic atheroma (15). MMP-1 is a highly specific protease capable of degrading insoluble fibrillar collagens (16), especially of types I and III, which are the major collagens of the atherosclerotic plaque (17). Because of the substrate specificity of MMP-1 and its colocalization with macrophages and degraded fibrillar collagen in the lesion (13), it has been hypothesized that this proteinase could contribute to the expansion and rupture of the plaque.

When fed a high-cholesterol, Western-type diet, the apoE knockout $(a p o E 0)$ mice $(18,19)$ rapidly develop complex atherosclerotic lesions that are very similar to those of humans $(20,21)$ but that do not rupture (22). Mice do not possess a homologous gene to human MMP-1 (23), and remodeling of the collagen matrix in mouse atherosclerosis would therefore be performed by collagenase-3 (MMP-13), which is known to be expressed in plaques $(24,25)$. A recently cloned MMP, Mcol-A (murine collagenase-like A), may represent the murine orthologue to human MMP-1, although its specific activity against type I collagen is significantly lower and its pattern of expression in disease is unknown (26). To establish the in vivo role of MMP-1 in atherosclerosis, we have generated a transgenic mouse model that specifically expresses human MMP- 1 in tissue macrophages (27). The effect of the transgenic expression of MMP-1 on aortic atherogenesis was examined in apoE0 mice. 


\section{Methods}

Generation of mice. ApoE0 mice on the C57BL/ 6 genetic background were obtained from The Jackson Laboratory (Bar Harbor, Maine, USA). The generation of the transgenic mice was described previously (27). Briefly, the sequences upstream from the translation initiation codon of the human MMP-1 gene were removed. The collagenase genomic fragment $(9.3 \mathrm{~kb})$ was inserted downstream of the human scavenger receptor A enhancer/promoter sequence $(4.5 \mathrm{~kb})$ using a newly created $S m a$ I restriction site (Figure 1). The transgene was isolated from the cloning plasmid by NotI/SalI digestion, purified by $\mathrm{CsCl}$ gradient centrifugation, and microinjected into fertilized mouse eggs (F1[C57BL/6xCBA/J]x F1[C57BL/6xCBA/J]). Mice were crossed into the $\mathrm{C} 57 \mathrm{BL} / 6$ background for more than six generations and then crossbred with apoE0 mice. The animals were genotyped by PCR and Southern blot analysis as described elsewhere (28). All characterization studies were done on a second line of mice, and the data are identical to those presented here.

$R$ Nase protection. Total RNA was prepared from mouse tissue and peritoneal macrophages using the guanidinium thiocyanate-cesium chloride method (29), and RNase protection analysis was performed as described previously (30). Ten micrograms of RNA from a transgenic mouse line that overexpressed MMP-1 in the lung (30) were hybridized as a positive control.

Western blot analysis. Detection of human MMP-1 protein was performed by semiquantitative Western blot analysis. Mice were injected intraperitoneally with $1 \mathrm{ml}$ of $4 \%$ thio- glycolate, and the ascites fluid collected after 4 days. The macrophages were centrifuged in $5 \mathrm{ml}$ of PBS, resuspended in $10 \mathrm{ml}$ of Neuman and Tytell serumless medium (Life Technologies Inc., Gaithersburg, Maryland, USA), and plated on a $150-\mathrm{mm}$ Petri dish. The medium was replaced after 2 hours of incubation at $37^{\circ} \mathrm{C}$, and the cells were then incubated for another 48 hours. The culture supernatant was concentrated 12 -fold. To analyze the proteins from the aorta, the tissue was homogenized in $2 \mathrm{ml}$ of PBS containing $1 \%$ Triton X-100, $0.1 \%$ SDS, $0.2 \% \mathrm{NaN}_{3}, 1 \mathrm{mM}$ EDTA, and serine- and cysteine-proteinases inhibitors (Complete Mini; Roche Molecular Biochemicals, Indianapolis, Indiana, USA). A bicinchoninic acid protein assay (Pierce Chemical Co., Rockford, Illinois, USA) was performed, to control for loading of protein for each sample on a SDS-PAGE (10\%). After migration, the proteins were transferred to a nitrocellulose membrane (BioRad Laboratories Inc., Hercules, California, USA). The primary antibody used was a polyclonal rabbit antibody to human MMP-1 (Chemicon International Inc., Temecula, California, USA). Blots were developed by chemiluminescence as described by the manufacturer (NEN Life Science Products Inc., Boston, Massachusetts, USA).

Collagenase assay. Qualitative levels of MMP-1 protein were determined by a collagenase gel assay, to detect specific collagen degradation fragments. The macrophages were collected as already described here and were cultured in Neuman and Tytell serumless medium (Life Technologies Inc.) overnight. The culture medium was collected and concentrated 12-fold. Activation of latent MMP-1 was per-

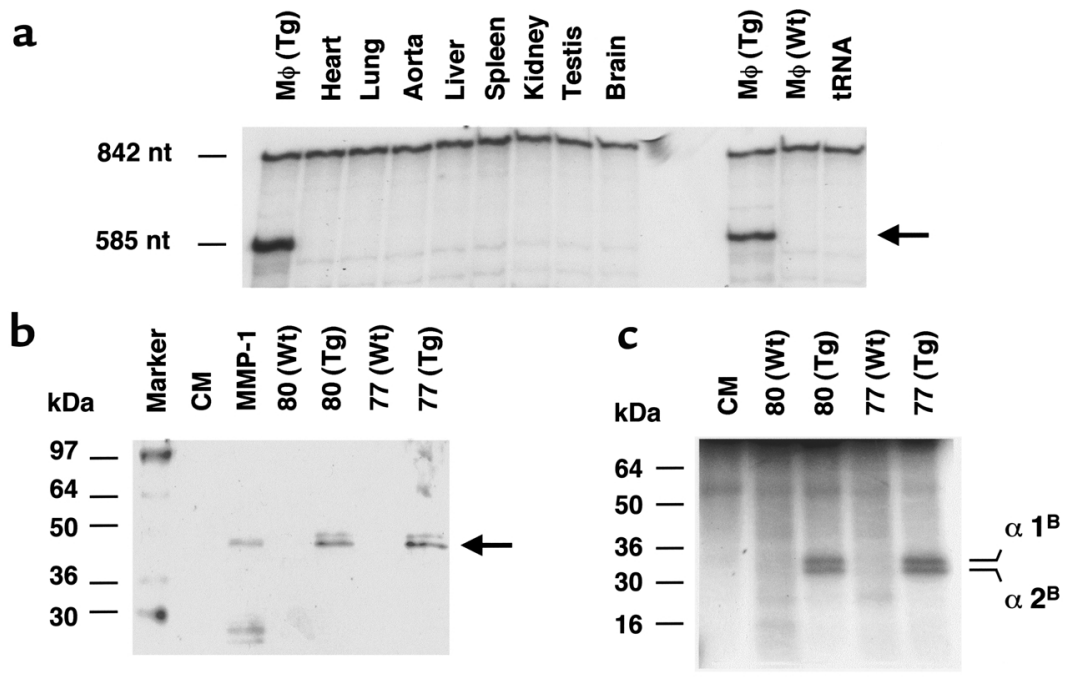

\section{Figure 1}

Expression of human MMP-1 in transgenic macrophages. (a) Tissue expression pattern of human MMP-1 in transgenic mice. An RNase protection assay was performed using RNA isolated from peritoneal macrophages (M $\phi)$, the heart, lung, aorta, liver, spleen, kidney, testis, and brain. Both wild-type $(\mathrm{Wt})$ and transgenic $(\mathrm{Tg})$ macrophages were analyzed. The unprotected probe is 842 nucleotides ( $\mathrm{nt}$ ), and the protected fragment is 585 nucleotides. (b) Western blot analysis of peritoneal macrophages culture media after activation with APMA. The activated human collagenase- $1\left(M_{r} 45,000\right)$ is detected only in the media from transgenic macrophages, not from wild-type. Macrophages from two transgenic and two wild-type mice were tested. Activated purified human interstitial collagenase (MMP-1) and culture medium alone (CM) were used as controls. (c) Collagenase activity in the culture media from transgenic and normal peritoneal macrophages. Culture media of peritoneal macrophages from two transgenic and two wild-type mice were activated with APMA and incubated with type I collagen. The characteristic $1 / 4 \mathrm{COOH}$-terminal fragments of the monomeric $\alpha 1$ and $\alpha 2$ chains from type I collagen, produced by MMP- 1 , are detected in the media from transgenic macrophages. 


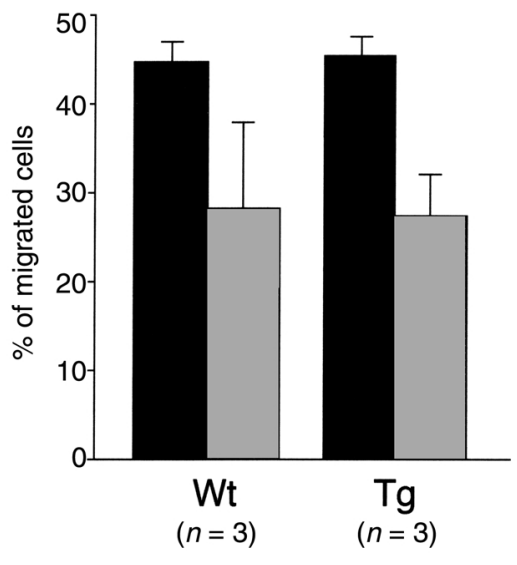

formed by incubation with $1.5 \mathrm{mM}$-aminophenylmercuric acetate (APMA; Sigma Chemical Co., St. Louis, Missouri, USA) for 12 hours at $37^{\circ} \mathrm{C}$. Ten microliters of each sample was incubated with $5 \mu \mathrm{l}$ of ${ }^{14} \mathrm{C}$-labeled rat tail type I collagen $(3 \mathrm{mg} / \mathrm{ml})(31)$ in a total volume of $20 \mu \mathrm{L}$ (50 $\mathrm{mM}$ Tris-HCl [pH 7.5], $0.15 \mathrm{M} \mathrm{NaCl}, 10 \mathrm{mM} \mathrm{CaCl}_{2}, 0.05 \%$ Brij 35, and $0.02 \% \mathrm{NaN}_{3}$ ) overnight at $37^{\circ} \mathrm{C}$. The digestion products were analyzed by SDS-PAGE (9\%).

Lipid and fast performance liquid chromatography analysis. Plasma samples from fasted apoE0 $(n=2)$ or transgenic apoE0/MMP-1 $(n=3)$ mice (on a Western diet for 16 weeks) were pooled to a final volume of $100 \mu \mathrm{l}$, and their lipoproteins were fractionated by fast performance liquid chromatography (FPLC) (Superose 6; Amersham Pharmacia Biotech Inc., Piscataway, New Jersey, USA). Phospholipids and total cholesterol levels of each fraction $(1.5 \mathrm{ml})$ were determined by a $4-$ aminoantipyrine-based enzymatic assay (Wako Bioproducts, Richmond, Virginia, USA).

Quantitative cell migration assay. Peritoneal macrophages $(250,000$ cells per well) were plated on Boyden chambers coated with human fibronectin or type I collagen (Chemicon International Inc.) in DMEM medium with $10 \%$ FBS. The lower compartment of the chambers contained the same medium with murine monocyte chemotactic protein-1 (MCP1) (R\&D Systems Inc., Minneapolis, Minnesota, USA) at $10 \mathrm{ng} / \mathrm{ml}$. After 48 hours of incubation $\left(37^{\circ} \mathrm{C}, 5 \% \mathrm{CO}_{2}\right)$, the number of macrophages having penetrated the gel were quantified following the manufacturer's instructions.

Histological analysis. Paraffin-embedded tissues were sectioned $(4 \mu \mathrm{m})$ and stained with hematoxylin and eosin (H\&E) for light microscopy. Serial sections were also stained by silver impregnation and Mallory trichrome, for collagen fibers, and Elastica van Gieson, for elastic fibers (32).
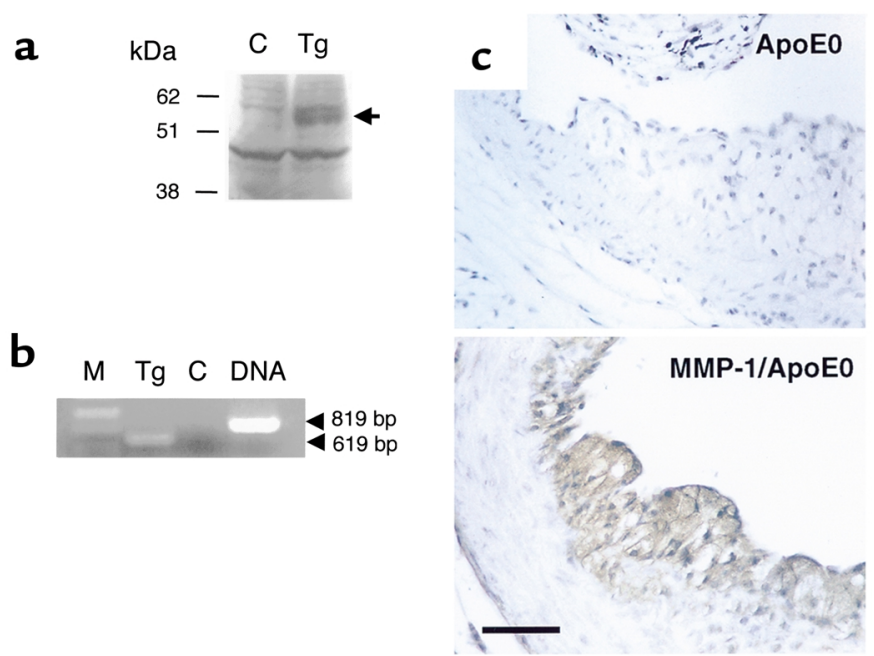

\section{Figure 3}

Presence of human MMP-1 in the atherosclerotic lesions of transgenic mice. (a) Western blot analysis of the aortic proteins from apoE0 (C) and transgenic MMP-1/apoE0 ( $\mathrm{Tg}$ ) mice using a rabbit polyclonal antibody against human MMP-1. Fifty micrograms of proteins were loaded on the gel. A signal of $M_{r} 55,000$ corresponding to MMP-1 is detected in the extract from the transgenic mouse. (b) RT-PCR. One microgram of total RNA isolated from the aorta of a transgenic MMP-1/apoE0 mouse was used for RT-PCR using primers designed from exons 9 and 10 of the human MMP-1 gene. The expected size of 619 bp was detected ( $\mathrm{Tg}$ ). No signal was detected when the reverse transcriptase was replaced with Taq polymerase (C). The PCR product from genomic DNA shows the expected size of $819 \mathrm{bp}$. M=molecular weight marker. (c) Immunohistochemical detection of MMP-1. Sections of the proximal aortas from apoE0 and transgenic MMP-1/apoE0 mice that were fed a Western diet for 16 weeks were incubated with a mouse mAb raised against human MMP-1. Scale bar: $50 \mu \mathrm{m}$. 


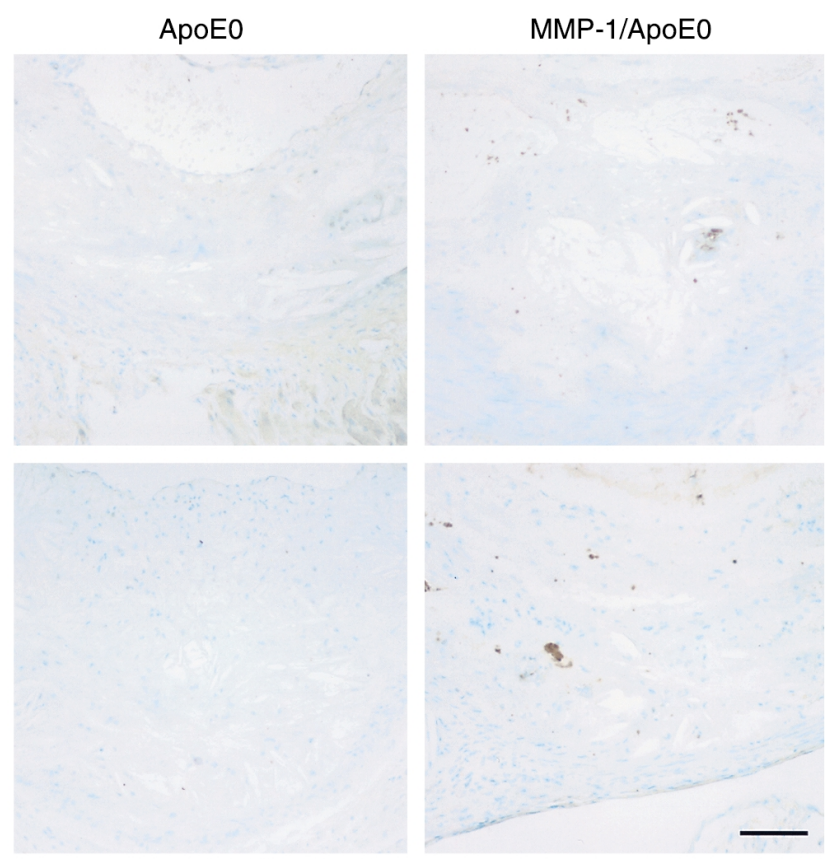

Figure 4

Immunohistochemical detection of cleaved type I collagen. Aortic sections from 30-week-old apoE0 and MMP-1/apoE0 mice were stained with the 9A4 mAb against collagenase-cleaved type I collagen. Sections were counterstained with methyl green. Scale bar: $100 \mu \mathrm{m}$.

Immunohistochemistry. Immunohistochemical detection of human MMP-1 in the lesions was performed using the avidin-biotin-horseradish peroxidase method (ScyTek, Logan, Utah, USA) with specific mouse monoclonal antibodies (Fuji Chemicals, Toyama, Japan) at a final concentration of $6 \mu \mathrm{g} / \mathrm{ml}$ in PBS. The activity of the peroxidase was revealed by diaminobenzidine as a substrate, yielding a brown deposit. Sections were counterstained with hematoxylin. The detection of cleaved type I collagen neoepitopes was performed with the biotinylated monoclonal 9A4 antibody (33) at a concentration of $20 \mu \mathrm{g} / \mathrm{ml}$ in PBS, followed by a streptavidin-linked peroxidase revelation system (Zymed Laboratories Inc., South San Francisco, California,
USA). Sections incubated with PBS alone were included as controls. Macrophages were detected using the rat anti-mouse Mac-3 monoclonal antibody (PharMingen, San Diego, California, USA).

Quantification of atherosclerosis. The mice were fed a high-fat Western-type diet (20\% protein, $50 \%$ carbohydrate, $21 \%$ fat, $0.21 \%$ cholesterol; Research Diets, New Brunswick, New Jersey, USA) for 16 weeks. They were anesthetized with $2.5 \%$ avertin intraperitoneally, the inferior vena cava was nicked and the heart was pressure-perfused at $80 \mathrm{mmHg}$ via left ventricular puncture. The heart was first perfused with PBS then with 10\% neutral buffer formalin for 5 minutes to fix the aorta. The tissue was embedded in paraffin for histological analysis or in OCT. compound (Tissue-Tek; Miles Laboratories, Elkhart, Indiana, USA) and snap-frozen for quantitation studies. Transverse sections of $10 \mu \mathrm{m}$ from the proximal aorta (covering a length of $1.2 \mathrm{~mm}$ ) were stained with oil red-O. Every eighth section, for a total of six sections, was quantitated for accumulation of intimal lipid by video microscopy using the Image Pro software (version 3.0; Media Cybernetics, Silver Spring, Maryland, USA), and an average value was determined for each mouse (34). For matrix quantification, five transverse sections from each proximal aorta were stained with Masson trichrome, and the collagen content was measured using Image Pro.

Statistical analysis. Analysis were performed by the unpaired Student's $t$ test, with $P<0.05$ considered significant. Lesion sizes are presented as mean \pm SEM.

\section{Results}

The human MMP-1 gene was placed under the control of the scavenger receptor A enhancer/promoter (27), which results in specific gene expression in tissue macrophages and foam cells of atherosclerotic lesions (35), and transgenic lines were established (27). The specificity of transgene expression was analyzed by RNase protection assay. Total RNA from resident peritoneal macrophages and from other tissues was tested, as shown in Figure 1a. MMP-1 expression was detected as a protected fragment of 585 nucleotides in the

\footnotetext{
Figure 5

Comparison of the aortic arch of apoE0 ( $\mathbf{a}$ and $\mathbf{c}$ ) and transgenic MMP-1/apoE0 (b and $\mathbf{d}$ ) mice after 16 weeks of the Western diet. Longitudinal sections were stained with H\&E (a and $\mathbf{b}$ ) and Mallory trichrome ( $\mathbf{c}$ and $\mathbf{d}$ ). The areas shown in $\mathbf{c}$ and $\mathbf{d}$ correspond to the black boxes shown in $\mathbf{a}$ and $\mathbf{b}$, respectively. Scale bar: (a and $\mathbf{b}$ ) $200 \mu \mathrm{m}$; (c and d) $50 \mu \mathrm{m}$.
}
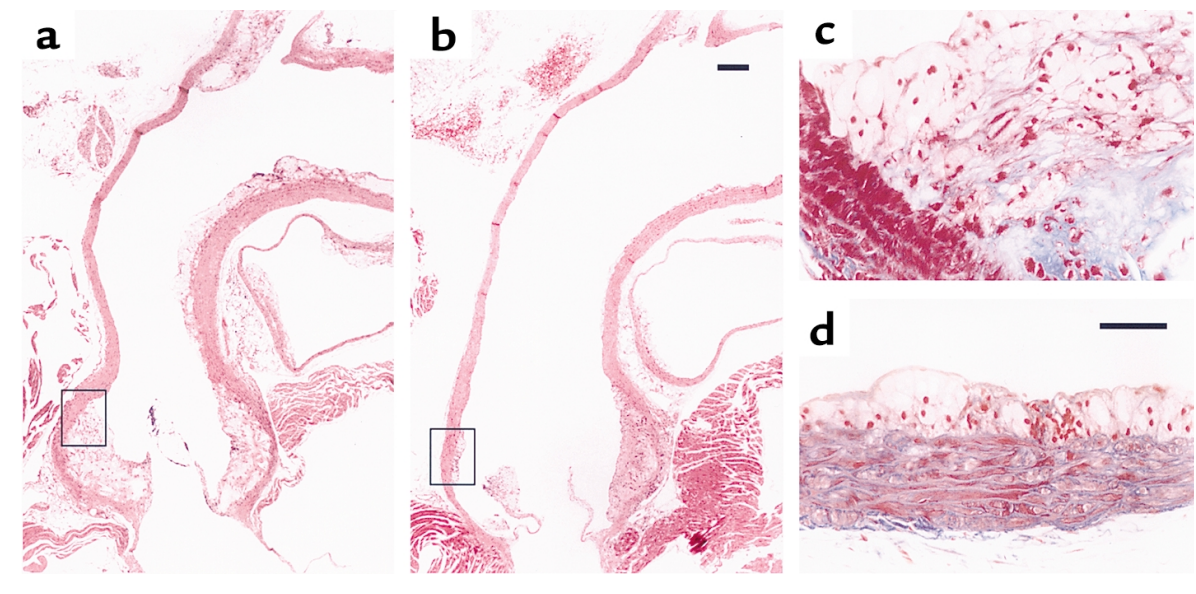


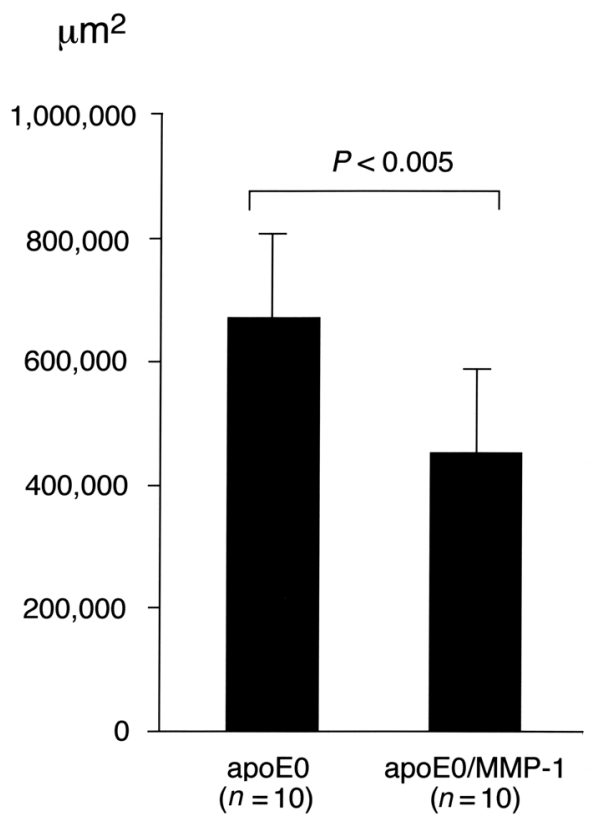

Figure 6

Quantitative comparison of the atherosclerotic lesions in apoE0 and transgenic MMP-1/apoE0 mice that were fed a Western diet for 16 weeks. Oil red-O-stained sections of proximal aortas from 21-weekold apoE0 $(n=10)$ and apoE0/MMP-1 $(n=10)$ were quantitated for neointimal lipid accumulation by video microscopy. Lesion sizes are presented as mean \pm SEM.

macrophages from two transgenic lines but not in the macrophages from wild-type littermates, nor in any of the other tissues analyzed.

The presence of secreted MMP-1 in the culture media of elicited peritoneal macrophages of MMP-1 transgenic mice was detected by Western blot analysis (Figure $1 \mathrm{~b}$ ). The APMA-activated MMP-1 (45 kDa) was detected in the culture media of transgenic macrophages, from transgenic lines 80 and 77 , but not in the media of the wild-type macrophages (Figure 1b). Interstitial collagenase specifically cleaves type I collagen at residues $775-776$, generating a $3 / 4 \mathrm{NH}_{2}$-terminal and a $1 / 4 \mathrm{COOH}$-terminal fragments (16). The characteristic collagenase digestion pattern on type I collagen was found in the APMA-activated media of macrophages from transgenic mouse lines 80 and 77, but not from wild-type lines (Figure 1c). We can conclude that this activity was due to the presence of the transgenic MMP-1, as no collagenase activity was detected in the culture media from normal macrophages. In addition, when transgenic macrophages were incubated with plasmin, MMP-1 activity could be detected in the culture medium, without requiring APMA activation. Culture medium from wild-type macrophages, even when incubated with plasmin, did not exhibit MMP-1 activity (data not shown).

To determine whether the transgene affected the migration and chemotaxis properties of the macrophages, their capacity to invade an immobilized ECM in vitro was test- ed (36). Macrophages from both groups of animals $(n=$ 3 ) migrated equally well in response to MCP-1 (Figure 2). Moreover, the number of cells collected from the peritoneum 5 days after thioglycolate injection was similar between the wild-type $(5.43 \pm 1.11$ million cells per milliliter, $n=3)$ and the transgenic mice $(5.36 \pm 1.24$ million cells per milliliter, $n=3$ ). The macrophages were identified by murine Mac-3 immunostaining followed by flow cytometry (data not shown) and represented approximately $75 \%$ of each cellular population.

To study the role of macrophage MMP- 1 in atherogenesis, the transgenic mouse line that expressed the highest level of human collagenase (line 77) (27) was crossed with the atherosclerosis susceptible apoE0 mice. A study population of apoE0 mice was generated that was heterozygous for the MMP-1 gene, and nontransgenic apoE0 littermates were used as the control group. The two groups were fed a Western-type diet for periods of 16-25 weeks. To determine whether the transgene affected the plasma lipoprotein levels of the mice, the cholesterol and phospholipid distribution in FPLC profiles of the two groups of animals was analyzed and found to be almost identical (data not shown). The total plasma cholesterol and triglyceride levels were also similar between the transgenic MMP1/apoE0 $(1,236.5 \pm 235.2 \mathrm{mg} / \mathrm{dl}$ and $93.3 \pm 20.9 \mathrm{mg} / \mathrm{dl}$, respectively; $n=6)$ and the control apoE0 $(1,117.0 \pm$ $416.4 \mathrm{mg} / \mathrm{dl}$ and $87.7 \pm 32.0 \mathrm{mg} / \mathrm{dl}$, respectively; $n=7$ ).

The in vivo expression of the transgene in the lesions was confirmed by Western blotting (Figure 3a) of aortic protein extracts and by RT-PCR of aortic RNA (Figure 3b). MMP-1 was also detected by immunohistochemistry using mAb's, with a signal in the thickened intima of the transgenic MMP-1/apoE0 mouse, but not in the control apoE0 (Figure 3c). Demonstration of MMP-1 activity in the lesions was performed by the identification of the specific degradation product of collagenase-cleaved type I collagen, using the biotinylated monoclonal antibody 9A4 (33). An increased signal in the lesions of transgenic MMP-1/apoE0 mice compared with apoE0 controls is shown in Figure 4.

After 16 weeks on the Western diet, aortas were fixed and a careful histological examination of the lesions was performed. The atheromatous lesions in the MMP-1/apoE0 mice were found to be less extensive and immature when compared with their littermate controls. Longitudinal sections of the aortic arches of transgenic MMP-1/apoE0 mice $(n=5)$ stained with $\mathrm{H} \& \mathrm{E}$ (Figure $5 \mathrm{~b}$ ) showed smaller supravalvular atheromatous plaques and minimal ascending aortic lesions when compared with the control apoE0 mice $(n=4)$ (Figure 5a). The lesions of the MMP-1 transgenic animals had less intimal thickening and longitudinal extent, especially in the ascending aorta and branch point. Mallory trichrome staining showed lipid-filled macrophages and cholesterol clefts between multiple layers of collagen fibers in the intimal lesions of control mice (Figure 5c). Intimal collagen staining was much less evident in the MMP-1 

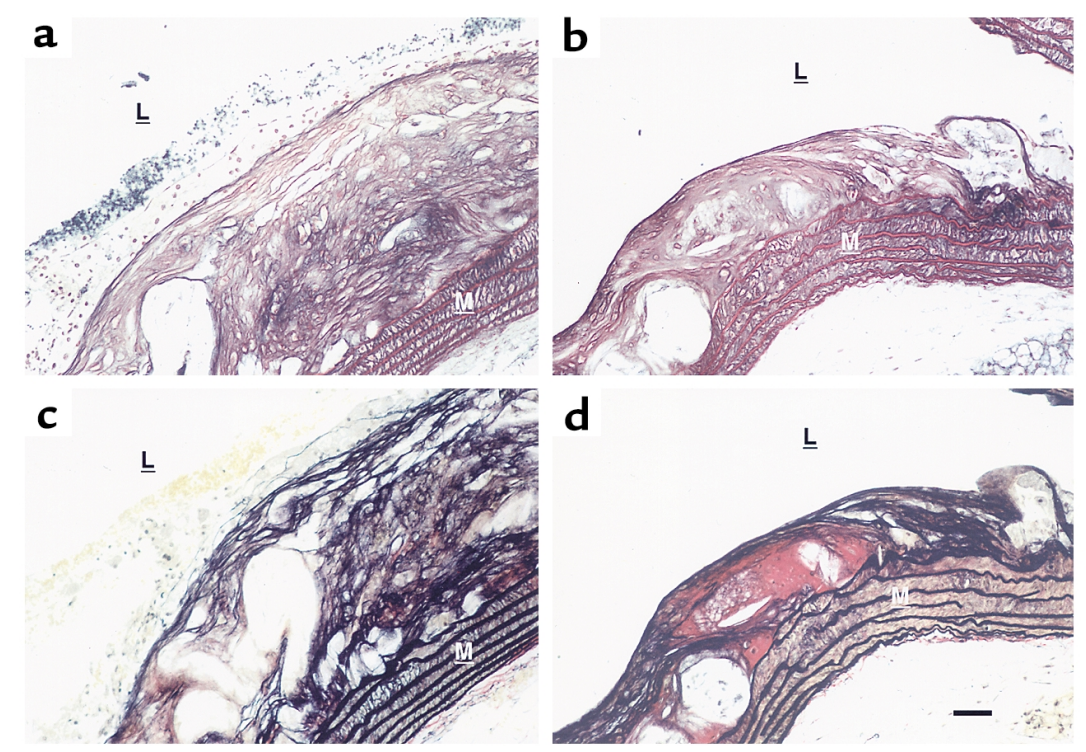

\section{Figure 7}

Comparison of atherosclerotic lesions after 25 weeks of the Western diet. Histological sections at the same level of the proximal aortas of apoE0 ( $\mathbf{a}$ and $\mathbf{b}$ ) and transgenic MMP-1/apoE0 (c and $\mathbf{d}$ ) mice were stained with silver impregnation (a and $\mathbf{c}$ ), for collagen fibers, and Elastica van Gieson ( $\mathbf{b}$ and $\mathbf{d}$ ), for elastin. The media (M) and the lumen ( $\mathrm{L})$ of the aorta are indicated. Blood cells can be seen on the top of the lesion. Aortas from three MMP-1/apoE0 and four apoE0 mice were examined. Scale bar: $50 \mu \mathrm{m}$.

transgenic mice (Figure 5d). At a comparable level, the atheromatous plaque of the transgenic mice presented a smaller and earlier type of lesion, generally described as fatty streaks (Figure $5 \mathrm{~d}$ ). Collagen was less abundant in this plaque compared with the control, and multiple strata were not identified.

The observed difference in lesion size was demonstrated through a quantitative atherosclerosis assay after 16 weeks of the atherogenic diet (Figure 6). This showed a significantly decreased average subintimal lipid deposition in the proximal aortas of transgenic MMP1/apoE0 mice $\left(459,134 \pm 150,351 \mu \mathrm{m}^{2} ; n=10\right)$ compared with the apoE0 controls $\left(673,470 \pm 139,868 \mu \mathrm{m}^{2}, n=10\right.$; $P<0.005$, Student's $t$ test). It is, however, notable that this assay, which only measures the lipid content, shows a less dramatic difference than the histological examination, which includes the connective tissue of the lesion. When the lesions were quantitatively examined for matrix content with Masson trichrome staining, a decrease in the average collagen content was found in the transgenic lesions when compared with the lesions from control animals: after 10 weeks of Western diet, 95,793 $\pm 35,720 \mu \mathrm{m}^{2}(n=5)$ and $146,760 \pm 33,851 \mu \mathrm{m}^{2}(n=4)$, respectively $(P=0.03)$; and after 16 weeks of Western diet, $54,113 \pm 38,792 \mu \mathrm{m}^{2}(n=3)$ and $155,956 \pm 67,176 \mu \mathrm{m}^{2}(n$ $=3$ ), respectively. Immunohistochemical analysis of the lesions showed no difference in macrophages (Mac3) and smooth muscle cells ( $\alpha$-actin) content between the two groups of animals (data not shown).

Further examination of the lesions was performed after 25 weeks of the atherogenic diet (Figure 7). Cross-sections of the vessel wall were stained by silver impregnation and Elastica van Gieson. In apoE0 control mice $(n=5)$, an abundant collagen matrix formed numerous layers throughout the thickness of the atheromatous lesions (Figure 7a). The adjacent media showed a rich network of matrix, surrounding smooth muscle cells between parallel elastic fibers (Figure $7 \mathrm{~b}$ ). In contrast, the smaller plaques in the MMP-1 transgenic mice $(n=3)$ had fewer cellular layers and a less abundant silver-stained collagen matrix (Figure 7c). Fewer layers of collagen and elastic fibers were present in those lesions, and where present, these layers did not span the full thickness of the plaque. In the control, a more prominent cellular layer is also apparent (Figure 7, a and b). Immunohistochemistry using specific antibodies for type I and III collagens, respectively, demonstrated a decreased intensity in the lesions of the transgenic mice (data not shown). There was no demonstrable difference in the rich network of collagen matrix surrounding the smooth muscle cells of the media of both groups of animals. No evidence of plaque rupture in the aortas of transgenic MMP-1/apoE0 mice was observed.

\section{Discussion}

The present study demonstrates that the in vivo expression of MMP-1 in the macrophages of apoE knockout mice significantly decreases atherosclerotic lesion formation. We initially undertook this study to address the hypothesis that overexpression of MMP-1 in mouse macrophages might lead to plaque rupture; surprisingly, we found no evidence of rupture in transgenic MMP-1/apoE0 mice lesions compared with control apoE0 mice. These data thus suggest a beneficial role of MMP- 1 in plaque progression, perhaps related to altered retention of atherogenic lipoproteins in plaques due to the change in matrix composition (37). Similar 
FPLC lipid-profiles and in vitro macrophage invasion capabilities between the two groups of mice demonstrate that the observed difference in the lesions was not due to variations in the plasma lipoprotein composition or to impaired migration properties of the transgenic macrophages.

We show here that peritoneal macrophages from normal mice do not have collagenolytic activity. Lack of collagenase activity in nonstimulated macrophages appears to be a characteristic of rodents (38), in contrast with human macrophages in culture that secrete MMP-1 and can degrade collagen from atherosclerotic fibrous caps (39). The lack of collagenolytic activity in rodent macrophages could account for some of the phenotypic differences between rodents and humans seen in macrophage-dependent disease processes (40).

Although the exact mechanism by which MMP-1 could delay atherogenesis in the transgenic mice is unknown, several hypotheses may account for this observation. The interaction of peripheral blood monocytes with the vascular matrix is important for their subsequent differentiation into resident lipid-laden macrophages. In particular, an interaction with type I collagen enhances the in vitro differentiation of monocytes into macrophages and increases their intracellular lipid accumulation (41). Digestion of type I collagen by MMP-1 could affect the differentiation of monocytes into macrophages and delay atherogenesis. The presence of MMP-1 could also decrease the infiltration of smooth muscle cells into the lesion, another crucial event in atherosclerosis development (42). The production of collagen by smooth muscle cells has been shown to be necessary for the normal migration of these cells in vitro (43), and degradation of this newly secreted collagen by MMP- 1 could impair the migratory process.

In vitro, type I and III fibrillar collagens bind native LDL and, more efficiently, oxidized LDL $(44,45)$. Smaller lesions in the transgenic mice could result from decreased neointimal retention of oxidized LDL, a highly atherogenic factor (1), owing to a smaller amount of intact fibrillar collagens in the plaques. Finally, the cleavage of an unknown physiological substrate of MMP-1, such as an inflammatory factor, could account for the observed phenotype.

Our data demonstrate that expression of MMP-1 during atherogenesis results in smaller, less advanced lesions with diminished collagen content, suggesting that increased degradation of the collagen matrix delays the progression of atherogenesis to the more severe stages. Although this finding was unexpected, the results are consistent with some earlier observations. The human stromelysin-1 (MMP-3) gene is differentially regulated by two common genetic variants of its promoter, with one variant leading to higher transcriptional activity (46). Patients with coronary atherosclerosis who are homozygous for the weaker promoter of stromelysin-1 developed a more rapid progression of the disease when compared with patients who were heterozygous, suggesting that a reduced expression of stromelysin- 1 is associated with increased lesion progression (46). Therefore we can hypothesize that remodeling of the ECM of atherosclerotic lesions by MMP- 1 and -3 has a beneficial role in atherosclerosis, by preventing or delaying the progression of the pathology.

Our study also demonstrates that expression of MMP-1 alone does not cause plaque rupture in apoE0 mice. This observation suggests that other proteases, acting alone or together with MMP-1, are needed to break the fibrous cap of lesions. Cysteine proteinases cathepsins $\mathrm{S}$ and $\mathrm{K}$ have been recently described in human atheroma (47), in conjunction with a deficiency of their potent inhibitor cystatin C (48). In vitro secretion of these cysteine-proteinases by human monocyte-derived macrophages has been shown to be an important source of proteolytic activity (49). In addition to MMPs, overexpression of active cysteineproteinases might thus play an important role in the matrix remodeling of the vascular wall during atherosclerosis and plaque rupture.

Factors other than proteinases contribute to the vulnerability of the lesions. This is suggested by the demonstration of the normal fibrous caps in the lesions of transgenic mice. Plaque rupture is a multifactorial process in which important parameters such as hemodynamic force, circumferential stress, and blood pressure $(2,3,50)$ are substantially different between mice and humans and could account for the lack of rupture seen in the apoE0 animals.

In conclusion, our results suggest the intriguing possibility that macrophage expression of MMP-1 in the human lesions of atherosclerosis has a beneficial role, preventing or delaying the development of the pathology. This is the first in vivo demonstration of a potential beneficial role for MMP-1 in disease pathology, which is most likely attributed to the function of this enzyme in remodeling and repair of the ECM rather then tissue destruction.

\section{Acknowledgments}

The authors thank Seema Dalal and Henry Kim (Columbia University) for their technical assistance, and Peter Mitchell (Pfizer, Groton, Connecticut) for the generous gift of the 9A4 antibody. J. D'Armiento is a recipient of a Burroughs Welcome Career Award. This work was supported by American Heart Association grant $9850025 \mathrm{~T}$ (J. D’Armiento) and in part by NIH grant HL56984 (J. D’Armiento).

\footnotetext{
1. Ross, R. 1999. Atherosclerosis: an inflammatory disease. N. Engl. J. Med. 340:115-126.

2. Shah, P.K. 1996. Pathophysiology of plaque rupture and the concept of plaque stabilization. Cardiol. Clin. 14:17-29.

3. Schroeder, A.P., and Falk, E. 1996. Pathophysiology and inflammatory aspects of plaque rupture. Cardiol. Clin. 14:211-220.

4. Libby, P. 1995. Molecular bases of the acute coronary syndromes. Circulation. 91:2844-2850.

5. Massova, I., Kotra, L.P., Fridman, R., and Mobashery, S. 1998. Matrix metalloproteinases: structures, evolution, and diversification. FASEB J. 12:1075-1095.

6. Galis, Z.S., Sukhova, G.K., Lark, M.W., and Libby, P. 1994. Increased
} 
expression of matrix metalloproteinases and matrix degrading activity in vulnerable regions of human atherosclerotic plaques. J. Clin. Invest. 94:2493-2503.

7. Nikkari, S.T., et al. 1995. Interstitial collagenase (MMP-1) expression in human carotid atherosclerosis. Circulation. 92:1393-1398.

8. Li, Z., et al. 1996. Increased expression of 72-kd type IV collagenase (MMP2) in human aortic atherosclerotic lesions. Am. J. Pathol. 148:121-128.

9. Brown, D.L., Hibbs, M.S., Kearney, M., Loushin, C., and Ismer, J.M. 1995 Identification of $92-\mathrm{kd}$ gelatinase in human coronary atherosclerotic lesions. Association of active enzyme synthesis with unstable angina. Circulation. 91:2125-2131.

10. McMillan, W.D., et al. 1995. In situ localization and quantification of mRNA for 92-kd type IV collagenase and its inhibitor in aneurysmal, occlusive, and normal aorta. Arterioscler. Thromb. Vasc. Biol. 15:1139-1144.

11. Henney, A.M., et al. 1991. Localization of stromelysin gene expression in atherosclerotic plaques by in situ hybridization. Proc. Natl. Acad. Sci. USA. 88:8154-8158.

12. Halpert, I., et al. 1996. Matrilysin is expressed by lipid-laden macrophages at sites of potential rupture in atherosclerotic lesions and localizes to areas of versican deposition, a proteoglycan substrate for the enzyme. Proc. Natl. Acad. Sci. USA. 93:9748-9753.

13. Sukhova, G.K., et al. 1999. Evidence for increased collagenolysis by interstitial collagenases- 1 and -3 in vulnerable human atheromatous plaques. Circulation. 99:2503-2509.

14. Rajavashisth, T.B., et al. 1999. Membrane type 1 matrix metalloproteinase expression in human atherosclerotic plaques. Evidence for activation by proinflammatory mediators. Circulation. 99:3103-3109.

15. Galis, Z.S., Sukhova, G.K., Kranzhoefer, R., Clark, S., and Libby, P. 1995. Macrophage foam cells from experimental atheroma constitutively produce matrix-degrading proteinases. Proc. Natl. Acad. Sci. USA. 92:402-406.

16. Jeffrey, J.J. 1998. Interstitial collagenases. In Matrix metalloproteinases. W.C. Parks and R.P. Mecham, editors. Academic Press Inc. San Diego, California, USA. $15-42$

17. Katsuda, S., et al. 1992. Collagens in human atherosclerosis. Immunohistochemical analysis using collagen type-specific antibodies. Arterioscler. Thromb. 12:494-502.

18. Plump, A.S., et al. 1992. Severe hypercholesterolemia and atherosclerosis in apolipoprotein E-deficient mice created by homologous recombination in ES cells. Cell. 71:343-353.

19. Zhang, S.H., Reddick, R.L., Piedrahita, J.A., and Maeda, N. 1992. Spontaneous hypercholesterolemia and arteria lesions in mice lacking apolipoprotein E. Science. 258:468-471.

20. Zhang, S.H., Reddick, R.L., Burkey, B., and Maeda, N. 1994. Diet-induced atherosclerosis in mice heterozygous and homozygous for apolipoprotein E gene disruption. J. Clin. Invest. 94:937-945.

21. Nakashima, Y., Plump, A.S., Raines, E.W., Breslow, J.L, and Ross, R. 1994. ApoE-deficient mice develop lesions of all phases of atherosclerosis throughout the arterial tree. Arterioscler. Thromb. Vasc. Biol. 14:133-140.

22. Breslow, J.L. 1996. Mouse models of atherosclerosis. Science. 272:685-688.

23. Vincenti, M.P., et al. 1998. Cloning of the gene for interstitial collagenase-3 (matrix metalloproteinase-13) from rabbit synovial fibroblasts: differential expression with collagenase- 1 (matrix metalloproteinase-1). Biochem. J. 331:341-346.

24. Carmeliet, P., et al. 1997. Urokinase-generated plasmin activates matrix metalloproteinases during aneurysm formation. Nat. Genet. 17:439-444.

25. Jeng, A.Y., et al. 1999. Enhanced expression of matrix metalloproteinase$3,-12$, and -13 mRNAs in the aortas of apolipoprotein E-deficient mice with advanced atherosclerosis. Ann. NY Acad. Sci. 878:555-562.

26. Balbin, M., et al. 2001. Identification and enzymatic characterization of two diverging murine counterparts of human interstitial collagenase (MMP-1) expressed at sites of embryo implantation. J. Biol. Chem. 29:319-322.

27. Lemaître, V., O’Byrne, T.K., Dalal, S.S., Tall, A.R., and D'Armiento, J. 1999. Macrophage-specific expression of human collagenase (MMP-1) in transgenic mice. Ann. NY Acad. Sci. 878:736-739.

28. Sambrook, J., Fritsch, E.F., and Maniatis, T. 1989. Molecular cloning: a lab oratory manual. Cold Spring Harbor Laboratory Press. Plainview, New
York, USA 9.31-9.59.

29. Chirgwin, J., Przybyla, A., McDonald, R., and Rutter, W.J. 1979. Isolation of biologically active ribonucleic acid from sources enriched in ribonuclease. Biochemistry. 18:5294-5299.

30. D’Armiento, J., Dalal, S.S., Okada, Y., Berg, R.A., and Chada, K. 1992. Collagenase expression in the lungs of transgenic mice causes pulmonary emphysema. Cell. 71:955-961.

31. Cawston, T.E., and Barrett, A. 1979. A rapid and reproducible assay for collagenase using $\left[1-{ }^{14} \mathrm{C}\right]$ acetylated collagen. Anal. Biochem. 99:340-345.

32. Putt, F. 1972. Manual of histopathological staining methods. John Wiley \& Sons. New York, New York, USA. 111-126.

33. Otterness, I.G., et al. 1999. Detection of collagenase-induced damage of collagen by 9A4, a monoclonal C-terminal neoepitope antibody. Matrix Biol. 18:331-341.

34. Gupta, S., et al. 1997. IFN-gamma potentiates atherosclerosis in apoE knock-out mice. J. Clin. Invest. 99:2752-2761.

35. Horvai, A., et al. 1995. Scavenger receptor A gene regulatory elements target gene expression to macrophages and foam cells of atherosclerotic lesions. Proc. Natl. Acad. Sci. USA. 92:5391-5395.

36. Shipley, J.M., Wesselschmidt, R.L., Kobayashi, D.K., Ley, T.J., and Shapiro, S.D. 1996. Metalloelastase is required for macrophage-mediated proteolysis and matrix invasion in mice. Proc. Natl. Acad. Sci. USA. 93:3942-3946.

37. Williams, K.J., and Tabas, I. 1995. The response-to-retention hypothesis of early atherogenesis. Arterioscler. Thromb. Vasc. Biol. 15:551-561.

38. Gibbs, D.F., Warner, R.L., Weiss, S.J., Johnson, K.J., and Varani, J. 1999. Characterization of matrix metalloproteinases produced by rat alveolar macrophages. Am. J. Respir. Cell Mol. Biol. 20:1136-1144.

39. Shah, P.K., et al. 1995. Human monocyte-derived macrophages induce collagen breakdown in fibrous caps of atherosclerotic plaques. Potential role of matrix-degrading metalloproteinases and implications for plaque rupture. Circulation. 92:1565-1569.

40. Gibbs, D.F., et al. 1999. Role of matrix metalloproteinases in models of macrophage-dependent acute lung injury. Evidence for alveolar macrophage as source of proteinases. Am. J. Respir. Cell Mol. Biol. 20:1145-1153.

41. Wesley, R.B., II, Meng, X., Godin, D., and Galis, Z.S. 1998. Extracellular matrix modulates macrophage functions characteristic to atheroma: collagen type I enhances acquisition of resident macrophage traits by human peripheral blood monocytes in vitro. Arterioscler. Thromb. Vasc. Biol. 18:432-440.

42. Schwartz, S.M. 1997. Smooth muscle migration in atherosclerosis and restenosis. J. Clin. Invest. 100(Suppl.):S87-S89.

43. Rocnik, E.F., Chan, B.M.C., and Pickering, G. 1998. Evidence for a role of collagen synthesis in arterial smooth muscle cell migration. J. Clin. Invest. 101:1889-1898.

44. Hoover, G.A., McCormick, S., and Kalant, N. 1988. Interaction of native and cell-modified low density lipoprotein with collagen gel. Arteriosclerosis. 8:525-534.

45. Jimi, S., Sakata, N., Matunaga, A., and Takebayashi, S. 1994. Low density lipoproteins bind more to type I and III collagens by negative chargedependent mechanisms than to type IV and V collagens. Atherosclerosis. 107:109-116.

46. Ye, S., et al. 1996. Progression of coronary atherosclerosis is associated with a common genetic variant of the human stromelysin-1 promoter which results in reduced gene expression. J. Biol. Chem. 271:13055-13060.

47. Sukhova, G.K., Shi, G.P., Simon, D.I., Chapman, H.A., and Libby, P. 1998. Expression of the elastolytic cathepsins $\mathrm{S}$ and $\mathrm{K}$ in human atheroma and regulation of their production in smooth muscle cells. J. Clin. Invest. 102:576-583.

48. Shi, G.P., et al. 1999. Cystatin C deficiency in human atherosclerosis and aortic aneurysms. J. Clin. Invest. 104:1191-1197.

49. Reddy, V.Y., Zhang, Q.Y., and Weiss, S.J. 1995. Pericellular mobilization of the tissue-destructive cysteine proteinases, cathepsins B, L, and S, by human monocyte-derived macrophages. Proc. Natl. Acad. Sci. USA. 92:3849-3853. 50. Falk, E. 1992. Why do plaques rupture? Circulation. 86(Suppl.):III30-III42. 